\section{Konkurrenz unter Radiologen - zur Anwendbarkeit des Wettbewerbsrechts zwischen Teilnehmern an der vertragsärztlichen Versorgung}

\section{Einleitung}

Das System der Gesetzlichen Krankenversicherung (GKV) ist getragen von dem Gedanken einer staatlich geregelten und solidarisch gesteuerten Versorgung. Die Einbindung von Leistungserbringern in das Vertragsarztrecht dient vor allem der staatlichen Aufgabe der Daseinsvorsorge. ${ }^{1}$ Die an der vertragsärztlichen Versorgung teilnehmenden Erbringer radiologischer Leistungen sind untereinander jedoch zudem Wettbewerber auf dem Markt für diese Leistungen.

Das Gesetz gegen den unlauteren Wettbewerb (UWG) dient dem Schutz vor unlauteren geschäftlichen Tätigkeiten und gleichzeitig dem Interesse der Allgemeinheit an einem unverfälschten Wettbewerb.

Ein niedergelassener Radiologe, der unlautere Geschäftspraktiken ausübt, könnte nach dem UWG von seinen Konkurrenten auf das Unterlassen der unlauteren Handlung, auf Schadenersatz und auch auf die Auskunftserteilung sensibler Daten in Anspruch genommen werden. Die Wettbewerbsregeln des UWG sind ihrer Funktion und Natur nach jedoch lediglich auf solche Märkte anzuwenden, die durch das dynamische Verhältnis von Angebot und Nachfrage bestimmt sind. Das System der GKV steht dazu in deutlichem Kontrast. ${ }^{2}$ Es ist deshalb fraglich, ob Teilnehmer an der vertragsärztlichen Versorgung gegen konkurrierende Kollegen aus Vorschriften gegen den unlauteren Wettbewerb vorgehen können.

1 Becker/Kingreen, Der Krankenkassenwettbewerb zwischen Sozial- und Wettbewerbsrecht - Zur geplanten Ausdehnung der Anwendung des GWB auf das Handeln der Krankenkassen, NZS 2010, 417 (419).

2 Stand und Perspektiven des Wettbewerbs im deutschen Krankenversicherungssystem, Sondergutachten der Monopolkommissiongemäß § 44 Abs. 1 Satz 4 GWB, S. 14, Rn. 14, abrufbar unter: http://www.asscompact.de/ sites/asscompact.de/files/Monopolkommission.pdf; letzter Aufruf 08.10.2018.
Die Vorschriften für eine wettbewerbsrechtliche Steuerung sind im Bereich der GKV prinzipiell geringer entwickelt, da ein Wettbewerb der Akteure des Gesundheitssystems kein primäres Ziel der GKV ist. Die Einbindung von Ärzten in das Vertragsarztrecht dient vielmehr der Erfüllung der staatlichen Aufgabe der Daseinsvorsorge. ${ }^{3}$ Trotz der öffentlich-rechtlichen Ausgestaltung des gesetzlichen Krankenversicherungssystems kann es aber durchaus Geschäftstätigkeiten geben, die vorrangig den wirtschaftlichen Zweck der Gewinnerzielung verfolgen und bei denen folglich eine Anwendbarkeit des Wettbewerbsrechts sinnvoll erscheint.

Soweit ein Leistungserbringer beispielsweise für die von ihm angebotenen Leistungen oder seine Qualifikationen wirbt, privatärztliche Leistungen anbietet oder Vergünstigungen im Zusammenhang mit seinen Leistungen verspricht und dabei gegen Berufsrecht oder das Heilmittelwerbegesetz (HWG) verstößt, kann er von konkurrierenden Leistungserbringern grundsätzlich auch nach den Vorschriften des UWG oder den allgemeinen Vorschriften des Bürgerlichen Gesetzbuches (BGB) in Anspruch genommen werden. ${ }^{4}$

Umstritten ist jedoch seit langem, welche Rechtsschutzmöglichkeiten konkurrierende Leistungserbringer haben, wenn ihre Rechtsbeziehungen als Teilnehmer an der vertragsärztlichen Versorgung betroffen sind. Diese Frage regelt $\S 69$ Sozialgesetzbuch Fünftes Buch (SGB V). Seit dem GKVGesundheitsreformgesetz 2000 (GKV-GRG 2000) vom 22.12.1999, ${ }^{5}$ in Kraft getreten zum 01.01.2000, sind die Rechtsbeziehungen der Krankenkassen zu sämtlichen Leistungserbringern, also den Krankenhäusern, Vertragsärzten, Apotheken und allen sonstigen nichtärztlichen Leistungserbringern,

\footnotetext{
3 Becker/Kingreen, ebd.

4 Köhler in Köhler/Bornkamm, Gesetz gegen den unlauteren Wettbewerb, 34. Aufl. 2016, $\S 3 a$ Rn. 1.179 ff. u. Rn. 1.218ff. m.w.N.

5 BGBI I 2626.
}

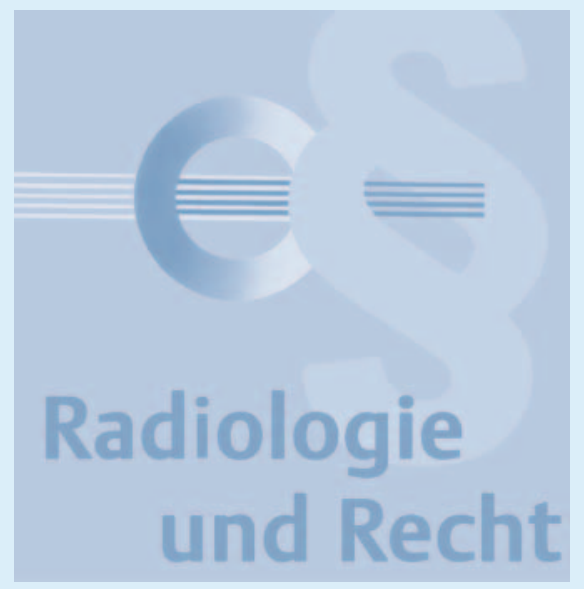

ausschließlich sozialversicherungsrechtlicher Natur und damit dem öffentlichen Recht zuzuordnen. ${ }^{6}$ Ob davon nach $\S 69$ Abs. 4 SGB V auch die Rechtsbeziehungen der an der vertragsärztlichen Versorgung teilnehmenden Leistungserbringer untereinander erfasst sind, war ebenfalls streitig.

Die Frage nach der Anwendbarkeit der Vorschriften des UWG auf die Rechtsbeziehungen zwischen Teilnehmern an der vertragsärztlichen Versorgung stand zuletzt im Mittelpunkt einer Entscheidung des Bundessozialgerichts (BSG), das sich mit Unterlassungs- und Auskunftsansprüchen eines Nephrologen zu befassen hatte, der sich u. a. gegen die einem Konkurrenten erteilte Genehmigung einer Nebenbetriebsstätte gewandt hatte. ${ }^{7}$

\section{Regelungsinhalt des $\S 69$ SGB V - keine unmittelbare Anwendung von UWG und BGB}

In der bezeichneten Entscheidung befasst sich das BSG zunächst mit der Frage, ob und wann eine unmittelbare Anwendung wettbewerbsrechtlicher Vorschriften im Falle von Streitigkeiten unter an der vertragsärztlichen Versorgung teilnehmenden Leistungserbringern überhaupt in Betracht kommt. Dazu stellt es unter Hinweis auf $\S 69$ Abs. 1 S. 1 SGB $V$ fest, dass die Rechtsbeziehungen der Krankenkassen und ihrer Verbände zu Ärz-

6 siehe auch: BSG, Urteil vom 02.07.2013, Az.: B 1 KR 49/12 R.

7 BSG, Urteil vom 15.03.2017, Az.: B 6 KA 35/ $16 R$. 
ten, Zahnärzten, Psychotherapeuten, Apotheken sowie sonstigen Leistungserbringern und ihren Verbänden einschließlich der Beschlüsse des Gemeinsamen Bundesausschusses und der Landesausschüsse nach den $§ \S 90$ bis 94 SGB V abschließend durch das Vierte Kapitel des SGB V sowie $§ \S 63,64$ SGB V geregelt werden. Es erklärt zudem ausdrücklich, dass die abschließende Regelung durch das Vierte Kapitel des SGB V sowie $\S \S 63,64$ SGB $\vee$ nach $\S 69$ Abs. 1 S. 4 SGB $\vee$ ebenfalls gelte, soweit die Rechtsbeziehungen Dritter, mithin auch diejenigen von vertragsärztlichen Leistungserbringern untereinander, betroffen sind. Zudem, so das BSG, gölten die Vorschriften des BGB gem. §69 Abs. 1 S. 3 SGB V ebenfalls nicht unmittelbar, sondern nur entsprechend und nur, soweit sie mit den Vorgaben und Pflichten der Beteiligten nach dem Vierten Kapitel des SGB V vereinbar sind.

Eine Anwendung des Wettbewerbsrechts könne darüber hinaus nicht damit begründet werden, dass Vertragsärzte nicht als Verwaltungshelfer der Krankenkassen oder der Kassenärztlichen Vereinigung tätig werden, sondern eine freiberufliche Tätigkeit ausüben. ${ }^{8}$ Das BSG stellt ausdrücklich klar, dass nach seiner Auffassung wegen der Regelungen in §69 SGB V die Anwendung des UWG auf die Beziehungen der Leistungserbringer untereinander stets ausgeschlossen ist, soweit Handlungen in Erfüllung des öffentlich-rechtlichen Versorgungsauftrags nach dem SGB V betroffen sind.

Schließlich, so das BSG, beziehe sich, soweit $\S 69$ Abs. 2 SGB V eine Ausnahme von dem Grundsatz des §69 Abs. 1 SGB V regele, diese Ausnahme ausdrücklich nur auf bestimmte, konkret bezeichnete Regelungen des Gesetzes gegen Wettbewerbsbeschränkungen (GWB) und nicht auf das UWG oder das BGB. Soweit §4 Abs. 3 S. 2 SGB V Unterlassungsansprüche und in diesem Zusammenhang eine entsprechende Anwendung von Vorschriften des UWG vorsehe, beziehe sich dies nicht auf das Verhältnis von Leistungserbringern untereinander, sondern allein auf das Verhältnis der Krankenkassen zueinander, und zwar beschränkt auf konkret bezeichnete Verfahrensvorschriften, vgl. §12 Abs. 1 bis 3

8 vgl. dazu BSG, Urteil vom 30.11.2016, Az.: B 6 KA 38/15R.
UWG. Wegen des klaren Wortlauts sei auch eine entsprechende Anwendung auf das Verhältnis von Leistungserbringern untereinander ausgeschlossen. Insgesamt, so das BSG weiter, komme daher eine unmittelbare Anwendung wettbewerbsrechtlicher Vorschriften im Falle von Streitigkeiten von an der vertragsärztlichen Versorgung teilnehmenden Leistungserbringern nicht in Betracht, wenn ihre Rechtsbeziehungen als Teilnehmer an der vertragsärztlichen Versorgung betroffen sind. §69 SGB V weise die entsprechenden Streitigkeiten zugleich dem Rechtsweg zu den Sozialgerichten zu.

\section{Anwendbarkeit wettbewerbsrechtlicher Grundsätze nur im Ausnahmefall}

Im Anschluss an diese Ausführungen beantwortet das BSG die Frage, ob nicht gleichwohl die mittelbare Heranziehung wettbewerbsrechtlicher Grundsätze regelmäßig gerechtfertigt sein kann, wenn die Rechtsbeziehungen von Teilnehmern an der vertragsärztlichen Versorgung betroffen sind und die fraglichen Handlungen die Erfüllung des öffentlich-rechtlichen Versorgungsauftrags nach dem SGB V betreffen.

Hierzu führt das BSG aus, dass es seit seinem Urteil vom 07.02.2007 ${ }^{9}$ in mehreren Entscheidungen geklärt habe, unter welchen Voraussetzungen Teilnehmer an der vertragsärztlichen Versorgung berechtigt sind, zugunsten ihrer Konkurrenten ergangene Entscheidungen der zuständigen Körperschaften bzw. Gremien der gemeinsamen Selbstverwaltung mit dem Ziel der Abwehr rechtswidriger Konkurrenz anzufechten. Damit sei eine Rechtfertigung für die Heranziehung wettbewerbsrechtlicher Grundsätze inzwischen jedenfalls soweit entfallen, wie eine Drittanfechtungsberechtigung in unmittelbarer Anwendung sozialrechtlicher Vorschriften besteht.

Anschließend befasst sich das BSG mit der Frage, ob auch in Fällen, in denen eine Drittanfechtungsberechtigung in unmittelbarer

9 BSG, Urteil vom 07.02.2007, Az.: B 6 KA 8/ $06 R$.
Anwendung sozialrechtlicher Vorschriften nicht besteht, eine entsprechende Anwendung wettbewerbsrechtlicher Grundsätze in Betracht kommt. Es verweist dazu auf ein Urteil aus dem Jahr 2011. ${ }^{10}$ In dieser Entscheidung war das BSG davon ausgegangen, dass allgemeine Schadenersatzregelungen auch für den Wettbewerb von Leistungserbringen untereinander gelten müssten und dass zu deren Konkretisierung die wettbewerbsrechtlichen Grundsätze des UWG heranzuziehen seien.

Ob an dieser Rechtsprechung überhaupt festzuhalten ist, lässt das BSG in dem hier behandelten Urteil zwar im Ergebnis offen. Es schränkt diese Rechtsprechung aber insoweit ein, als es eine entsprechende Anwendung der wettbewerbsrechtlichen Grundsätze des UWG nur dann für gerechtfertigt hält, wenn ansonsten keine anderweitigen primären Rechtsschutzmöglichkeiten bestehen. Eine entsprechende Anwendung der Vorschriften des UWG zu Unterlassungs-, Schadenersatz- und Auskunftsansprüchen auf die Rechtsbeziehungen von Leistungserbringern nach dem SGB V untereinander komme, so das BSG, nur in Betracht, soweit dies ausnahmsweise zur Vermeidung verfassungsrechtlich nicht hinnehmbarer Rechtsschutzdefizite erforderlich ist.

Unterlassungs-, Schadenersatz- und Auskunftsansprüche der Teilnehmer an der vertragsärztlichen Versorgung untereinander kommen nach Auffassung des BSG somit allenfalls noch in ganz besonders gelagerten Fällen in Betracht, in denen es aus systematischen Gründen an verfassungsrechtlich zu fordernden primären Rechtsschutzmöglichkeiten fehlt, weil die zuständigen Gremien der vertragsärztlichen Versorgung nicht mit dem Ziel der Verhinderung rechtswidriger Konkurrenz in Anspruch genommen werden können, obwohl ein Verstoß gegen solche Vorschriften, die auch dem klagenden Arzt zu dienen bestimmt sind, in Rede steht.

Sei dies der Fall, könne dem allerdings nicht entgegengehalten werden, dass die Kassenärztlichen Vereinigungen verpflichtet sind, gegen die Verletzung vertragsärztlicher Pflichten mit Mitteln des Disziplinarrechts vorzugehen. Das Gebot effektiven Rechts-

10 BSG, Urteil vom 23.03.2011, Az.: B 6 KA 11/ $10 \mathrm{R}$. 
schutzes fordere, dass der Rechtsschutzbedürftige selbst die Möglichkeit haben muss, die gerichtliche Überprüfung der Inhalte der Entscheidung, die er anfechtet, zu erreichen.

\section{Effektiver Rechtsschutz gewährleistet?}

Fraglich bleibt nach der Entscheidung des BSG, ob ein Teilnehmer an der vertragsärztlichen Versorgung ausreichend effektiven Rechtsschutz gegenüber seinen ebenfalls vertragsärztlich tätigen Konkurrenten erlangen kann, soweit Handlungen in Erfüllung des öffentlich-rechtlichen Versorgungsauftrags nach dem SGB V betroffen sind.

Zum einen kennen die primären Rechtsschutzmöglichkeiten nach sozialrechtlichen Vorschriften bei Verletzung z. B. vertragsarztrechtlicher Vorschriften keine Unterlassungs-, Schadenersatz- oder Auskunftsansprüche der Teilnehmer an der vertragsärztlichen Versorgung untereinander. Soweit also die Anfechtung einer zugunsten eines Konkurrenten ergangenen Entscheidung der zuständigen Körperschaften bzw. Gremien der gemeinsamen Selbstverwaltung nach wettbewerbsrechtlichen Grundsätzen Unterlassungs-, Schadenersatz- oder Auskunftsansprüche des Anfechtenden begründen könnte, kann dieses Ziel nach der von dem BSG vertretenen Auffassung aufgrund der abschließenden Regelungen in §69 SGB V nicht erreicht werden. Zudem sind Fallgestaltungen, in denen die vom BSG in Erwägung gezogene entsprechende Anwendung wettbewerbsrechtlicher Grundsätze zur Gewährung verfassungsrechtlich gebotenen Rechtsschutzes in Betracht kommen könnte, nicht ersichtlich. Soweit Unterlassungs-, Schadenersatz- oder Auskunftsansprüche in entsprechender Anwendung des UWG geltend gemacht werden, setzt dies eine unlautere geschäftliche Handlung des Konkurrenten voraus. Kann sich dieser aber in einem sozialgerichtlichen Verfahren auf eine Entscheidung der zuständigen Körperschaften bzw. Gremien der gemeinsamen Selbstverwaltung berufen, wird eine unlautere geschäftliche Handlung seitens des Konkurrenten regelmäßig nicht anzunehmen sein.

Zum anderen erfordern wettbewerbsrechtliche Ansprüche regelmäßig eine sehr schnelle Geltendmachung im Wege des Eilrechts- schutzes. Ist die Entscheidung der zuständigen Körperschaften bzw. Gremien der gemeinsamen Selbstverwaltung durch den Konkurrenten erst einmal umgesetzt, z. B. die Tätigkeit aufgenommen, kommt gerichtlicher Rechtsschutz aus Sicht des Anfechtenden praktisch zu spät. Diesem Umstand wird vor den Zivilgerichten durch eine sehr schnelle Bearbeitung von Eilrechtsfahren in wettbewerbsrechtlichen Streitigkeiten Rechnung getragen. Die Dauer eines sozialgerichtlichen Verfahrens überschreitet diejenige eines Wettbewerbsprozesses im Zivilverfahren auch im Falle der Inanspruchnahme von Eilrechtsschutz regelmäßig deutlich. Die Zuweisung von Streitigkeiten über Handlungen in Erfüllung des öffentlich-rechtlichen Versorgungsauftrags nach dem SGB V zu den Sozialgerichten, die das BSG aufgrund des $§ 69$ SGB V annimmt, führt deshalb zu Rechtsschutzdefiziten, soweit wettbewerbsrechtliche Unterlassungs-, Schadenersatz oder Auskunftsansprüche in Betracht kommen können.

Schließlich, so wird vertreten, ${ }^{11}$ falle das Vertragsarztrecht grundsätzlich in den Anwendungsbereich der Richtlinie 2005/29/EG über unlautere Geschäftspraxen von Unternehmen gegenüber Verbrauchern im Binnenmarkt (UGP-RL), auch soweit der Wettbewerb von Leistungserbringern untereinander betroffen ist. Das Urteil des BSG könne daher zu einer richtlinienwidrigen Beschränkung des Konkurrenzschutzes der Teilnehmer an der vertragsärztlichen Versorgung auf den nur beschränkt wirksamen Primärrechtsschutz im Vertragsarztrecht führen, wenn die Überlegungen des BSG auf den Anwendungsbereich der UGP-RL übertragen würden.

\section{Wettbewerbsrecht bei privatärztlicher Tätigkeit}

Von dem Konkurrenzverhältnis von Vertragsärzten untereinander, soweit Handlungen in Erfüllung des öffentlich-rechtlichen Versorgungsauftrages nach dem SGB $\checkmark$ betroffen sind, abzugrenzen ist das Wettbewerbsverhältnis untereinander im Bereich der sonstigen ärztlichen Tätigkeit, das zivilrechtlich zu beurteilen ist.

11 vgl. Schreiber, Vertragsarztwettbewerb im lauterkeitsrechtsfreien Raum?, ZESAR 2018, $167 \mathrm{ff}$.
In diesen Fällen finden die Vorschriften des SGB V keine Anwendung. Ein Rechtsschutz nach sozialrechtlichen Vorschriften ist bei derartigen Rechtsverstößen durch Konkurrenten nicht zu erlangen. Der Rechtsschutz der Wettbewerbsteilnehmer untereinander muss sich deshalb nach den Vorschriften des UWG und des BGB richten. Die Vorschriften des HWG und der Berufsordnungen dienen dabei u. a. dem fairen Wettbewerb.

Das gilt beispielsweise für den $§ 31$ der Musterberufsordnung Ärzte (MBO-Ä). Dieser verbietet es Ärztinnen und Ärzten für die Zuweisung von Patientinnen und Patienten oder Untersuchungsmaterial oder für die Verordnung oder den Bezug von Arzneioder Hilfsmitteln oder Medizinprodukten ein Entgelt oder andere Vorteile zu fordern, sich oder Dritten versprechen oder gewähren zu lassen oder selbst zu versprechen oder zu gewähren. Die Vorschrift verbietet berufsrechtlich z. B. so genannte KickBack-Modelle bei der Zuweisung von Privatpatienten. Die Vorschrift dient dabei nicht nur dem Schutz des Patienten davor, dass der Arzt seine Behandlungsentscheidung möglicherweise nicht nur aus medizinischen, sondern auch aus monetären Gesichtspunkten trifft. Sie soll darüber hinaus verhindern, dass sich Ärzte durch die Gewährung ungerechtfertigter Vorteile Wettbewerbsvorteile gegenüber ihren Wettbewerbern verschaffen. ${ }^{12}$ Verstöße gegen die Vorschrift können deshalb im Verhältnis der Wettbewerber untereinander nach wettbewerbsrechtlichen Regelungen verfolgt werden. ${ }^{13}$ Es ist deshalb unter dem Gesichtspunkt der unangemessenen unsachlichen Einflussnahme gemäß § 1 UWG unlauter, einen Arzt durch die Gewährung oder das Inaussichtstellen eines finanziellen Vorteils zu veranlassen, diese Interessenwahrungspflicht zu verletzen. ${ }^{14}$

Der Bereich der ärztlichen Werbung ist ebenfalls in den Berufsordnungen der Ärzte

12 Ratzel in Ratzel/Lippert, Kommentar zur Musterberufsordnung der deutschen Ärzte, 6. Aufl. 2015, S.468.

13 Köhler, a.a.O., §3a Rn. 1.132 m.w.N.

14 vgl. BGH, Urteil vom 30.01.2003, Az.: I ZR 142/00; Urteil vom 21.04.2005, Az.: I ZR 201/ 02; Urteil vom 24.06.2010, Az.: I ZR 182/08; Urteil vom 09.09.2010, Az.: I ZR 157/08; Urteil vom 23.02.2012, Az.: I ZR 231/10. 
geregelt (vgl. § 27 MBO-Ä). Die Zulässigkeit von Werbung für konkrete Behandlungen oder Heilmittel richtet sich nach dem HWG. Wirbt ein Arzt z. B. in berufswidriger Weise oder in nach dem HWG unzulässiger Weise für bestimmte von ihm angebotene Behandlungen, kann dies ebenfalls eine Haftung gegenüber Mitbewerbern nach dem UWG begründen. ${ }^{15}$

\section{Fazit}

Das BSG hat in der zuvor behandelten Entscheidung die Anwendbarkeit des Wettbewerbsrechts zwischen Teilnehmern an der vertragsärztlichen Versorgung ausgeschlossen, soweit eine Anfechtungsberechtigung in unmittelbarer Anwendung sozialrechtlicher Vorschriften besteht. Selbst wenn eine solche Anfechtungsberechtigung nicht bestehe, sei die Heranziehung wettbewerbsrechtlicher Grundsätze nur in Ausnahmefällen gerechtfertigt. Zudem sieht das BSG die Streitigkeiten zwischen Teilnehmern an der vertragsärztlichen Versorgung, soweit Handlungen in Erfüllung des öffentlichrechtlichen Versorgungsauftrags nach dem SGB $V$ betroffen sind, den Sozialgerichten zugewiesen.

Die Durchsetzung wettbewerbsrechtlicher Unterlassungs-, Schadenersatz- und Auskunftsansprüche unter konkurrierenden Teilnehmern an der vertragsärztlichen Versorgung ist damit durch das BSG deutlich eingeschränkt und erschwert worden. Ist ein Teilnehmer an der vertragsärztlichen Versorgung durch die Handlung eines Konkurrenten betroffen und möchte die Unterlassung dieser Handlung, Schadenersatz oder eine Auskunfterteilung verlangen, ist genau zu prüfen, nach welchen Regelungen und auf welchem Rechtsweg er Rechtsschutz gegenüber dem Konkurrenten erlangen kann.

Soweit von dem vermeintlich rechtswidrigen Verhalten des Konkurrenten der öffent- lich-rechtliche Versorgungsauftrag nach dem SGB V nicht betroffen ist, kommt allein die Inanspruchnahme von Rechtsschutz nach den Vorschriften des UWG bzw. des $B G B$ in Betracht und ist der Weg zu den Zivilgerichten zu wählen.

Prof. Dr. Peter Wigge

Rechtsanwalt

Fachanwalt für Medizinrecht

Jonas Kaufhold

Rechtsanwalt

Annika Stöbener

Wissenschaftliche Mitarbeiterin

Rechtsanwälte Wigge

Scharnhorststraße 40

48151 Münster

Tel.: (0251) $53595-0$

Fax: (0251) 53595 - 99

Email: kanzlei@ra-wigge.de

www.ra-wigge.de 